\title{
Restoring touch
}

\author{
Medical professionals and robotics engineers count on materials scientists for the development of \\ electronic skins with lifelike tactile sensing capabilities.
}

The sense of touch plays a central role in shaping our interactions with the world - it helps us to distinguish pain, recognize objects and convey feelings and emotions. Patients using artificial limbs would more readily accept their prostheses if these were able to restore the sensory capabilities of human skin ${ }^{1}$ without loss of function. Robotics engineers likewise rely on skin-like tactile feedback as a tool to improve robots' dexterity and ability to sense their surroundings; the ability to recognize physical contact and respond accordingly is essential to establish natural interactions between humans and robots. Our skin, however, is a sophisticated network of receptors responding to multiple mechanical and thermal stimuli, while being elastic and resilient against impacts such complexity cannot be perfectly replicated by an artificial equivalent. However, progress in sensing technologies and flexible electronics has already led to the realization of simpler electronic prototypes that have effectively provided prosthetic limbs and robotic arms with a rudimentary sense of touch.

On page 937 of this issue of Nature Materials, Zhenan Bao and her colleagues review the state of the art of electronic skin, focusing on prosthetic applications. Describing the various transduction mechanisms currently explored in labscale pressure sensors, they show that the sensitivity of such devices is now on a par with that of human receptors. However, to be usable in prosthetics these sensors need to be realized on substrates that conform to the curves of the body and adapt to its movements without altering their performance, or breaking. Materials scientists and device engineers are putting forward several strategies to address this flexibility problem, including intrinsically stretchable electronic circuits based on insulating elastomers filled with conducting carbon materials or organic polymers, and ultrathin devices able to withstand bending and stretching.

To avoid imposing on users the burden of frequent and expensive replacements, electronic skin needs also to be resistant to 'wear and tear'. Similarly, mechanical durability is a mandatory requirement in robotics, as explained by Giorgio Metta

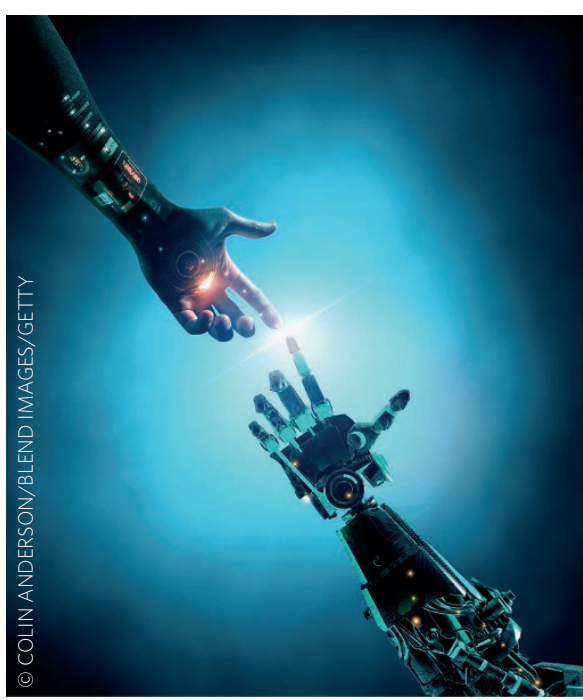

and high-capacity communication channel between the electronic circuitry and the neural tissues; the practical realization of this ideal brain/machine interface is still an open issue.

Several bioelectronic interfaces between rigid electrodes and the nervous system have demonstrated their clinical potential in modulating neural activity. For instance, probes for deep brain stimulation are being used for the treatment of tremors and Parkinson's disease ${ }^{2}$. However, extending such approaches to thousands of implanted needles - required to simultaneously excite multiple neural circuits and transmit the large amount of sensory information collected by the electronic skin - may not be feasible, as it would increase the risk of damaging the neural tissues or producing foreign-body reactions.

and his collaborators in a Commentary on page 921 . They describe a few implementations of flexible modules embedded with pressure sensors as well as proximity and temperature detectors that have been used to allow robots to recognize and manipulate objects. However, most of these systems still rely on dated technologies and off-the-shelf components, rather than the more sensitive but less robust prototypes demonstrated in research labs. Stepping up from the proofof-concept level requires the development of materials and device architectures with high sensitivity, without compromising on long term reliability.

The realization of a full-body-scale electronic skin poses further challenges related to the management of data received by thousands of different sensors. Solutions explored in robotics comprise electronic circuits that pre-process the signal at the single-sensor or singlemodule level, thus decreasing the amount of information to be transmitted to the central processing unit. In prosthetics applications, transmission of these data to the brain requires two additional steps. The first one is the translation of the sensors' output signal to a language intelligible to our neurons, which is now reliably implemented by specialized electronic circuits that modify the signal into waveforms resembling action potentials. The second requirement is a durable, fast Soft implants ${ }^{3-5}$ may better match the mechanical properties of the biological tissues and reduce the risk of implant rejection. An alternative to electrical stimulation is provided by optogenetics, a technique utilizing the excitation of genetically modified neurons using light pulses. The high selectivity and spatial resolution of this approach may allow the simultaneous delivery of multiple streams of data, reducing the number of required implants. Proof-of-concept devices embedding multiple LEDs or optical fibres in both rigid and soft implants have been successfully demonstrated ${ }^{6}$; however, the clinical translation of this genetics-based technology is proceeding at a slow pace, due to both technical and regulatory issues.

It is clear that several barriers mechanical compliance, long-term reliability, biological compatibility, increased device density and efficient data management - still need to be overcome for electronic skin to recreate lifelike tactile sensations in medical and robotics applications. Materials scientists are working hard to unlock this potential.

\footnotetext{
References

1. Kwok, R. Nature 497, 176-189 (2013).

2. Kringelbach, M. L., Jenkinson, N., Owen, S. L. F. \& Aziz, T. Z.

Nature Rev. Neurosci. 8, 623-635 (2007)

. Minev, I. R. et al. Science 347, 159-163 (2015).

4. Xie, C. et al. Nature Mater. 14, 1286-1292 (2015)

5. Yu, K. J. et al. Nature Mater. 15, 782-791 (2016).

6. Grosenick, L., Marshel, J. H. \& Deisseroth, K. Neuron 86, 106-139 (2015)
} 